\title{
ANÁLISIS
}

\section{BIBLIOTECAS PÚBLICAS EN COLOMBIA: TERRITORIO DE PAZ}

\author{
Public libraries in Colombia: Land of peace
}

\section{Orlanda Jaramillo}

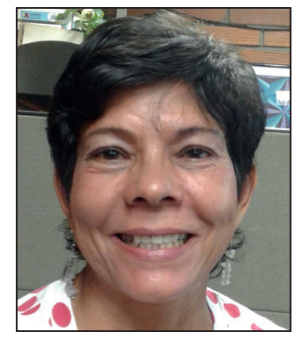

Orlanda Jaramillo es profesora titular e investigadora adscrita a la Escuela Interamericana de Bibliotecología de la Universidad de Antioquia. Bibliotecóloga, especialista en gerencia del desarrollo social de la Universidad Eafit y doctora en educación de la Universidad de Antioquia. Sus áreas de interés profesional son: biblioteca pública, educación en bibliotecología y procesos de autevaluación en educación superior.

http://orcid.org/0000-0002-3271-0256
Universidad de Antioquia Escuela Interamericana de Bibliotecología Calle 67 53-108. Bloque 12-336 Medellín (Antioquia), Colombia orlanda.jaramillo@udea.edu.co

\section{Resumen}

El fortalecimiento de la biblioteca pública como lugar de inclusión y cohesión social, de construcción de ciudadanía y memoria local, hace parte de los desafíos que se avizoran en su contribución para que el proceso de paz que enfrenta Colombia resulte tangible y pertinente. En este sentido se destacan algunas acciones que la entidad emprende para impulsar procesos de transformación social y, concretamente, para apoyar la construcción de tejido social en un momento histórico del país: el posconflicto. Para tal efecto se hace un acercamiento a su responsabilidad social para continuar con un recorrido por los procesos que han buscado finiquitar el conflicto armado, y terminar con el planteamiento de la biblioteca pública como territorio de paz, donde el bibliotecario se reinventa, aúna esfuerzos, emprende iniciativas y sueña. Más allá de la legislación y la normatividad, el posconflicto requiere instituciones y líderes que se comprometan y materialicen los cambios que surjan de las negociaciones de La Habana.

\section{Palabras clave}

Biblioteca pública; Procesos de paz; Recuperación de la memoria social; Ciudadanía; Inclusión social; Desarrollo de la comunidad; Participación ciudadana; Procesos sociales; Conflictos sociales; Colombia.

\begin{abstract}
The public libraries of Columbia need to situate themselves as places for inclusion, social cohesion, construction of citizenship, and local memory as part of the post-conflict peace process. Colombia's leaders and institutions, including libraries, have an obligation to go beyond the laws and regulations to achieve the vision of the future that has emerged from the negotiations in Havana, Cuba. There are actions that can be undertaken by libraries to promote social transformation at this historic time. Libraries are territories of peace, where librarians can undertake initiatives that build on citizens' dreams of a prosperous future.
\end{abstract}

\section{Keywords}

Public library; Peace building; Recovery of social memory; Social inclusion; Citizenship; Community development; Citizen participation; Social development; Social conflict; Colombia.

Jaramillo, Orlanda (2016). "Bibliotecas públicas en Colombia: territorio de paz". El profesional de la información, v. 25, n. 5, pp. 815-821. 
La biblioteca pública actúa como fuerza viva de la educación, cultura e información y como agente esencial del fomento de la paz y los valores espirituales en la mente del ser humano

(Unesco, 1994).

\section{Introducción}

Históricamente se ha reconocido el protagonismo de la biblioteca pública -en adelante BP- en la gestión, desarrollo y fortalecimiento de procesos de transformación social - sujetos y realidades sociales-, en tanto crea condiciones favorables para la inclusión y cohesión social, el diálogo y la recuperación de la memoria local, desde el acceso a la información, las TIC, el conocimiento, la educación y la cultura. Y con ello el diseño de programas, servicios y estrategias para lograr el desarrollo social con equidad.

Además se la valora como espacio vital, lugar para estar, para el refugio, en donde confluyen los integrantes de una comunidad sin condicionamiento alguno. Como institución, juega un papel fundamental en los propósitos de incrementar el capital social, potenciar la lucha contra la exclusión y promover la cohesión e inclusión social desde prácticas sociales, culturales y educativas que contribuyan al bienestar de las personas. Estas características han preparado y fortalecido a muchas bibliotecas públicas colombianas para responder a las demandas del actual proceso de posconflicto.

\section{Punto de partida: de templo del saber a} lugar de inclusión, diálogo y recuperación de la memoria

La aportación de la BP a procesos de transformación social se puede concretar a partir de la integración de dos componentes básicos:

- lo público, lo que pertenece a todos, por lo tanto es responsabilidad del Estado;

- lo político, en la orientación de su quehacer: cómo pensar la inclusión, la participación, el diálogo y el ejercicio ciudadano.
Estos componentes permiten el despliegue de los derechos y deberes que se originan en el ser social, en el reconocimiento de la alteridad $u$ otredad que interpela, limita y enriquece al sujeto como protagonista de las políticas, y a la BP como lugar de encuentro, de inclusión y cohesión social, de diálogo y memoria local -espacio vital dinamizador de procesos de transformaciones sociales- que crea las condiciones para que el usuario apropie y movilice información que le permita afrontar situaciones de su vida personal y de la comunidad. Así, la apuesta de la BP como espacio vital requiere centrar su atención en comunidades con alta vulnerabilidad social, víctimas del conflicto armado y que crean contextos emergentes donde ella interviene con procesos de sociabilidad -integración y adaptación- y de socialización - capacidad para relacionarse, interactuar en su entorno- (Jaramillo, 2010).

La BP contribuirá a los procesos de socialización, diálogo y recuperación de la memoria y búsqueda de mecanismos que permitan mitigar el dolor, devolver la esperanza y disminuir las cifras de la violencia

Sociabilidad y socialización desde donde se instauran los procesos de inclusión y cohesión social, al afianzar vínculos solidarios, recíprocos y de acogida, que posibilitan la reconstrucción del tejido social y cultural:

- la inclusión social es el conjunto de procesos que aseguran la integración de las personas en la vida comunitaria, especialmente de las que reúnen condiciones de vulnerabilidad social;

- la cohesión social se basa en el sentido de pertenencia, identidad, conexión y visión común que existe entre las personas y las comunidades dentro de una sociedad.

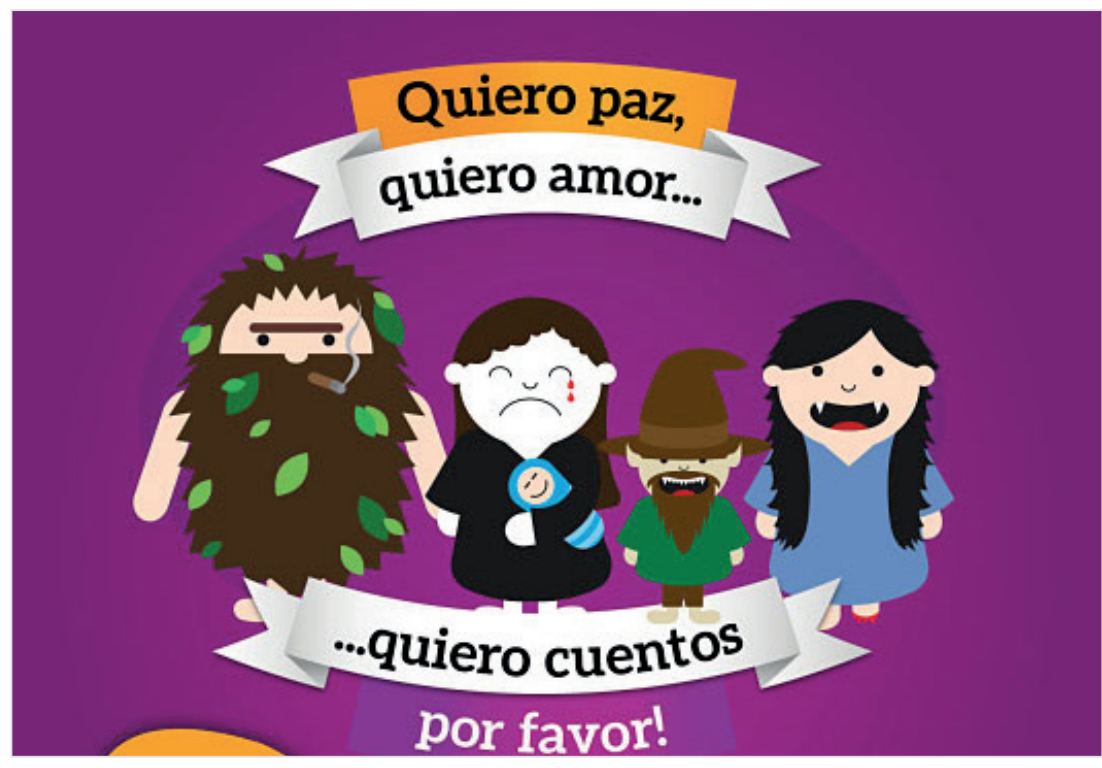

http://www.bibliotecanacional.gov.co/rnbp/noticias/quiero-paz-quiero-amor-quiero-cuentospor-favor-1
Por misión y definición, las BP son instrumentos de inclusión y cohesión social, pues sus actividades están destinadas a favorecer a todos los ciudadanos sin distinción alguna, en consonancia con lo estipulado en el Manifiesto de la Unesco (1994), con la generación de servicios, programas y espacios de confianza y convivencia, fundamentadas en el aprendizaje y la formación a lo largo de la vida.

Las BP son espacios donde el diálogo, es decir el encuentro cara a cara, se ejercita dentro de un ambiente seguro. Las partes dialogantes asumen una comprometida actitud de escucha y de respecto entre ellas para comunicarse, conocerse y generar diálogo y confianza como mecanismos de prevención, gestión y transformación de conflictos y de promoción y potenciación de una 
cultura de paz, logros que se fundamentan en procesos de recolección, organización y difusión de la memoria local, utilizando diversos medios, formatos y soportes.

\section{Contexto de los procesos de paz}

Los esfuerzos por consolidar un Estado-nación no han resultado suficientes para poner fin a los complejos conflictos sociales y económicos en los que surgen y actúan diferentes grupos insurgentes y contrainsurgentes. En palabras de Arias:

“En Colombia no se presentó un pacto de paz global sino un acumulado de procesos. Por eso ha sido un proceso de paz complejo, en varios episodios, con avances parciales valiosos, pero también con retrocesos. Con acciones propias de un posconflicto como son la Constitución Política de 1991, la reparación de las víctimas y la reintegración de excombatientes, pero a la vez con vigencia del conflicto bélico. No hay una continuidad lineal, hay períodos históricos con dinámicas determinadas y coyunturas diferenciadas.

La de los años 60 y 70 fue una guerra marginal, con prácticas degradadas y sordas, sin ningún diálogo. En los 80 se intensificaron diversas violencias y se expandieron las guerrillas, pero al adoptar el gobierno Betancur una política de paz se produjo el primer proceso de paz, en el que FARC, EPL y $M-19$ suscribieron treguas bilaterales e indefinidas con el Gobierno y se pactaron compromisos de reformas. Sin embargo, los acuerdos en gran medida no se aplicaron, la fuerza pública no acató la tregua y a los tres años todas las guerrillas habían retornado a la guerra. Al inicio de los 90 se acordó la paz con parte importante de la insurgencia. Su modelo, su logro y su gran consecuencia fue la Asamblea Nacional Constituyente de 1991 (...). El EPL propuso la Constituyente desde una tregua anterior a la que se había comprometido e insistía en que esta era la base para un cambio democrático que permitiera el paso a la acción política de toda la insurgencia. El M-19 pactó la paz sobre la base de una reforma constitucional en el Congreso, que, al no conseguirse, impulsó la Constituyente desde la legalidad, y agrupaciones armadas menores, como el PRT y el Movimiento Armado Quintín Lame, firmaron pactos de paz en el contexto de la Constituyente. Pero quedaron por fuera el $E L N$ y las FARC; no se consiguió la participación de las FARC y el ELN en la Constituyente del 91 por varias razones, entre ellas, el recrudecimiento de la ofensiva militar gubernamental y la consiguiente réplica guerrillera, asumiendo cada uno un cálculo en relación con un resultado exitoso en lo estratégico que se podía lograr por esa vía (Arias, 2015).

Arias advierte que se deben analizar las experiencias de diálogos de paz con las guerrillas que se cumplieron durante los períodos presidenciales 1998-2002 y 2002-2010; y otros factores políticos que favorecieron la reciente negociación de La Habana. Se trata de esfuerzos que pretenden finiquitar el conflicto armado y, según algunos expertos, generar acuerdos que lleven al país a esforzarse por garantizar los derechos de los colombianos a través de proyectos y programas de bienestar económico y social. Pero la paz sólo puede ser posible si el acuerdo firmado el 26 de septiembre de 2016 en la ciudad de Cartagena se materializa en procesos sociales, con especial influjo en las zonas más apartadas, escenarios de un conflicto armado estimulado por la ausencia del Estado. Justamente, una de las instituciones que contribuye a los procesos sociales y a la construcción de un nuevo país es la BP, dados su potencial y características que contribuirán a los procesos de socialización, diálogo y recuperación de la memoria y búsqueda de mecanismos que permitan mitigar el dolor, devolver la esperanza y disminuir las cifras de la violencia.

Colombia cuenta con 1.254 BP situadas en 1.125 municipios. El $87 \%$ se ubica en zonas urbanas y un $10 \%$ en parajes alejados. Una biblioteca por 35.509 habitantes y un promedio de 0,8 libros por habitante

\section{Puntos de encuentro: las bibliotecas públicas en los procesos de paz}

Las BP en Colombia ${ }^{1}$ están diseminadas por todo el territorio, pero las de mayor capacidad y mejor dotación se concentran en cinco departamentos: Antioquia, Boyacá, Cundinamarca, Santander y Valle, y concretamente en las ciudades capitales y en Bogotá como distrito capital; lo que quiere decir que el $87 \%$ se ubica en zonas urbanas y un $10 \%$ en parajes alejados.

Además de la dotación y ubicación, su trabajo se considera una actividad humana social, ciudadana y, en consecuencia, políticamente esencial en beneficio de la sociedad y del Estado (Meneses-Tello, 2013).

De acuerdo con los datos de la Red Nacional de Bibliotecas Públicas, el país cuenta con 1.254 BP situadas en 1.125 municipios. Con una correspondencia de acuerdo con los datos de la Red Nacional de Bibliotecas Públicas de una biblioteca por 35.509 habitantes y con un promedio de 0,8 libros por habitante, que disfrutan de sus servicios libre y gratuitamente.

Se trata del principal medio a disposición de la población colombiana para acceder a la información, el conocimiento y la cultura, en correspondencia con el propósito del gobierno nacional que a partir de 1968 instaura el Sistema Nacional de Bibliotecas Públicas. Años más tarde (1978) crea la Red Nacional de Bibliotecas Públicas - en adelante RNBP-como estrategia para hacer de Colombia un país lector ${ }^{2}$ y que cada municipio cuente siquiera con una biblioteca pública, al tiempo que diseña en el 2003 el Plan Nacional de Lectura y Bibliotecas (PNLB).

http://www.bibliotecanacional.gov.co/rnbp/directorio-debibliotecas-publicas

Aunque la distribución, infraestructura física, equipamiento, recursos bibliográficos y personal resultan poco idóneos debido a su aislamiento en relación con la centralidad del país, estas características no impiden que las BP hagan las veces de lugares de interrelaciones, intercambios, articula- 


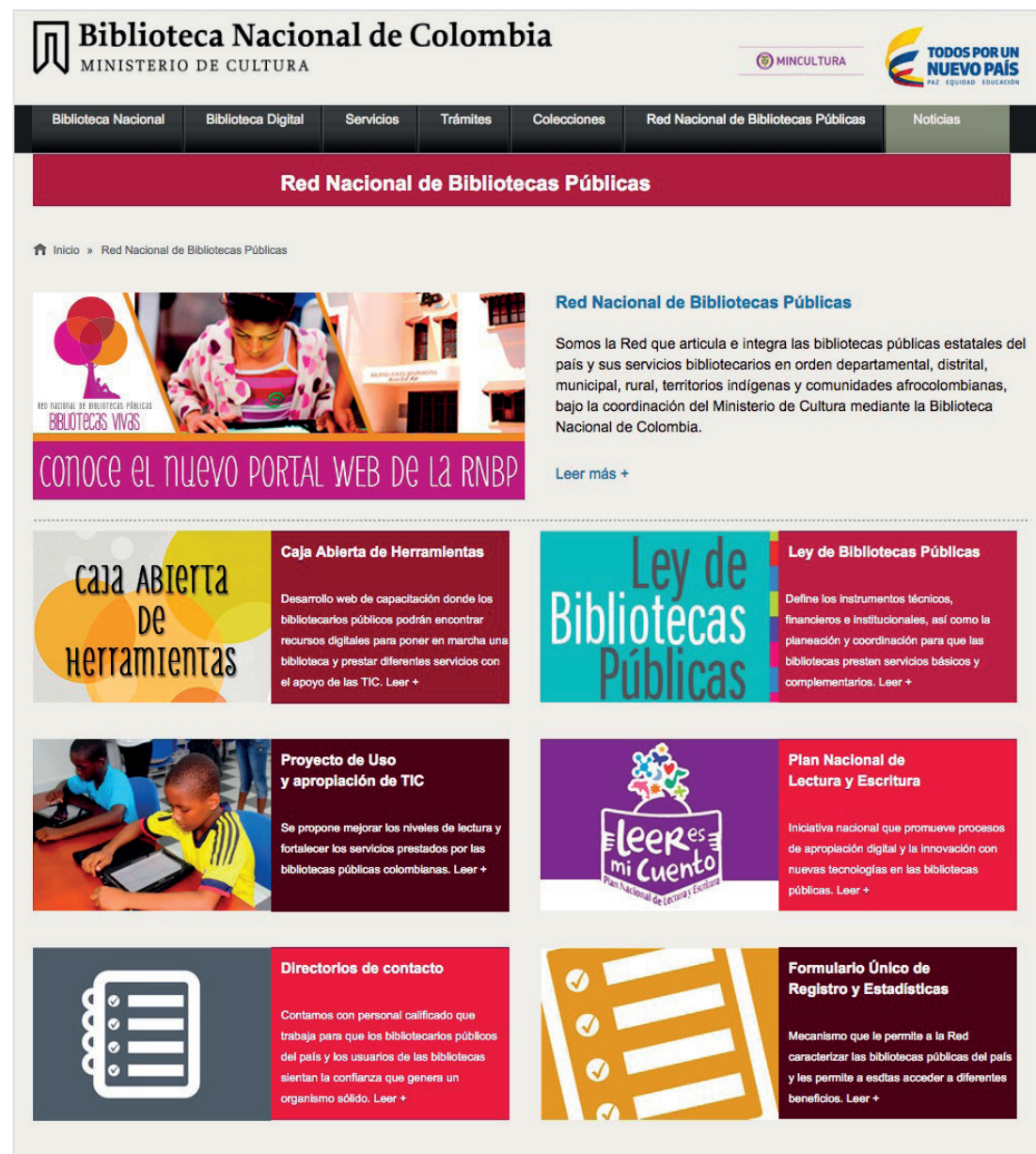

Red Nacional de Bibliotecas Públicas

http://www.bibliotecanacional.gov.co/content/red-nacional-de-bibliotecas-públicas

de contenidos, preservadora de la información y de la cultura, garante de acceso libre y equitativo a la información $(C C B, 2013)$; a partir de la oferta de servicios tradicionales - para facilitar su acceso a la información y la cultura-, pero también de servicios y acciones formativas que incorporen la alfabetización informacional, digital y mediática para paliar los efectos de la brecha digital y facilitar la inclusión cultural, social y laboral de los usuarios (Sánchez-García; Yubero, 2016, p. 229). Esta incorporación facilita la prestación de servicios sociales en formato electrónico disponibles todas las horas y días del año, y desde la comodidad de otros espacios diferentes a la biblioteca. Las TIC son las nuevas comunidades virtuales, espacios de expresión social, de la economía, la política y la cultura. Se trata de una búsqueda para vehiculizar las demandas y necesidades de la comunidad, a las cuales responde con estrategias mediadas por el respeto, la confianza, el buen trato y el reconocimiento. A la vez, ayuda a dignificar las personas, recuperar la esperanza en el entorno social y resarcir sus vidas, mientras posibilita el surgimiento de otra actitud frente a la vida, al entorno y a la sociedad, y con ella la inclusión, el diálogo y la recuperación de la memoria: ofrece espacios diferentes a los

ciones y de desarrollo humano, donde no sólo se apropia información y conocimiento sino que también se crean y re-crean vínculos y actos de transformación del sujeto y del colectivo (Jaramillo, 2010). En muchos casos se convierten en zonas neutrales frente al conflicto, que dan albergue a la población civil para que se entretejan las dimensiones de la memoria, la cultura, la conciencia, la voluntad y la utopía; dimensiones que expresan la apropiación que hacen de lo social y dan sentido a la historia.

Esta apertura del espacio permite que la comunidad aprenda a conocer, a hacer, a vivir unida y a ser, en la configuración de ciudadanos protagonistas de su propia realidad, capaces de definir sus proyectos de vida, de gestionar y decidir cada uno de los aspectos que atraviesan la cotidianidad. Así, la BP trasciende el concepto de depósito de libros, templo del saber y lugar para los escolares, para convertirse en parte activa y sensible de la comunidad y en agente de cambio a partir de nuevos modos de relación con los vecinos, que reaviva el compromiso cívico, impulsa la participación ciudadana y fomenta un mayor grado de intervención en los procesos de decisión y solución de los problemas de la comunidad (McDermott, 2010), conjugando elementos clave en la construcción de valores ciudadanos para alcanzar la igualdad de oportunidades en una sociedad incluyente.

Es la consideración de la BP como lugar estratégico en la comunidad, punto de encuentro, mediadora y proveedora espacios del conflicto, como se puede apreciar en el siguiente testimonio.

"La biblioteca ha servido para mejorar las condiciones del sector, tienen a dónde ir y cosas qué hacer; ha disminuido la violencia porque la gente se entretiene en la biblioteca y los niños y jóvenes no están tanto tiempo en la calle; los problemas de violencia han disminuido en el sector (...). Lo mejor de la biblioteca es que nos aleja de la continua guerra que se vive en la calle, es un lugar muy libre que da la oportunidad de aprender (...). Algo muy bonito es que a la sala internet llegan todos, de distintos barrios y grupos, ellos no van armados, llegan con los hijos y la esposa; ellos se sienten seguros y ven seguridad para los hijos (citado por Jaramillo y Quiroz, 2013).

Expresiones que permiten determinar a las BP como agentes clave en los procesos de inclusión, diálogo y memoria para algunos denominados como procesos posconflicto-, de donde deriva la complejidad de generar condiciones que favorezcan la inclusión social, que beneficien a los individuos y a los grupos; y el papel que las BP juegan en los procesos de paz, para que su trabajo sea una vía de inclusión, de diálogo, de desarrollo humano, de recuperación de la memoria local. Por ello, es imprescindible poner el acento en el valor de la biblioteca como agente de cohesión social, con una atención especial en los colectivos vulnerables y con necesi- 
dades específicas, como es el caso de las poblaciones de la mayoría de los municipios colombianos.

En el aporte de la BP a los procesos de paz merecen mención especial las acciones que en el último quinquenio desarrolla la RNBP y el PNLB: Leer es mi cuento, a partir de dos estrategias fundamentales:

- el Programa de promotores y tutores;

- el Premio nacional de bibliotecas públicas Daniel Samper Ortega.

Estas estrategias pretenden consolidar las bibliotecas públicas como escenarios de paz.

http://www.bibliotecanacional.gov.co/rnbp

\subsection{Programa de promotores, tutores y mentores}

El Programa de tutores, promotores y mentores se desarroIla a partir de visitas periódicas de expertos en BP y en lectura, a los bibliotecarios y las administraciones locales de los 32 departamentos del país que forman parte de la RNBP. Estas asesorías buscan fortalecer, implementar y mejorar los servicios y la gestión integral de las BP. El objetivo es recuperar las visiones y vivencias que sobre el conflicto armado manejan muchos colombianos, con el propósito de consolidar las BP como escenarios de paz, como mediadoras en procesos de reconciliación, para apuntarle a la reconstrucción del tejido social a partir de la palabra, la cultura y el reconocimiento de la diferencia.

Es imprescindible poner el acento en el valor de la biblioteca como agente de cohesión social, con una atención especial en los colectivos vulnerables

\subsection{Premio nacional de bibliotecas públicas Daniel Samper Ortega}

Lo organiza el Ministerio de Cultura y la Biblioteca Nacional de Colombia, y se enmarca en el PNLB que busca aumentar los índices de lectura en el país - de 1,9 a 3,2 en 2018-. Para lograrlo, el Ministerio de Cultura se propone construir BP en sectores donde residen comunidades pobres y que han resultado víctimas de la violencia, aumentar la oferta y el acceso a los libros e incrementar y mantener actualizadas las colecciones en las bibliotecas públicas y conectarlas de manera eficiente a internet.

http://www.bibliotecanacional.gov.co/rnbp

En su primera convocatoria (2014) el Premio buscaba reconocer el trabajo de las BP en todo el país, resaltando la importancia de su quehacer en la vida de las comunidades, el fomento de la lectura y la promoción de estándares de calidad en la gestión bibliotecaria. Pero también, sensibilizar a la opinión pública en torno al papel que cumplen como escenarios para el desarrollo de los municipios y el fomento de la lectura en las regiones del país.

Por estas razones la postulación debía resaltar aquellos méritos de la BP relacionados con el impacto positivo de los servicios y los programas o las acciones que incidieran en el desarrollo de la comunidad y en la mejora de sus condiciones de vida. En consecuencia, para determinar los ganadores se tuvieron en cuenta:

- experiencias de las bibliotecas;

- liderazgo de los bibliotecarios;

- condiciones en que desempeñan su labor;

- papel que cumple la institución como escenario para el rescate de la memoria;

- trabajo con jóvenes, niños y población vulnerable.

El Premio fue otorgado a la Biblioteca Pública Municipal Luis Carlos Galán Sarmiento, del Valle del Guamuez, en el departamento de Putumayo. A continuación se presentan testimonios acerca de la biblioteca ganadora:

"Nuestra idea es que desde la biblioteca se impulse el diálogo y sigamos construyendo la paz a partir de la lectura y el diálogo, porque gracias a esas lecturas realizadas desde la infancia es que comienzan a transmitirse esos lazos de solidaridad y de amistad para reconstruir el tejido social" (...)

"Se trataba de continuar funcionando pese a tener que desenvolvernos en medio de un contexto violento, con el fin de poder aportar la paz desde una dinámica local que nos permitiera hacer frente a la violencia a través de estrategias tan sutiles como la de convocar a los niños a ciclos de cine foro; desarrollar tertulias literarias; abrir convocatorias de cuentos en torno a la vida en el municipio, o contar con la posibilidad de tener un programa radial dedicado a la música, el radio teatro o la poesía", explica un integrante del Grupo de Amigos de la Biblioteca (...)

"Los actores armados no paran muchas bolas a ese tipo de cuestiones ligadas a la literatura o el arte en general, porque consideran que se trata de actividades que en nada pueden contribuir a cambiar el curso del conflicto" (...)

"Aunque la guerrilla prohibía que cualquier tipo de institución estatal se acercara a estas regiones, la biblioteca nunca llegó a ser vetada, puesto que tenía una connotación cultural y de hacer llegar el conocimiento a poblaciones muy apartadas. De tal suerte que nuestra presencia cobró un valor muy significativo entre los campesinos, en la medida que los niños podían contar con un nuevo espacio de esparcimiento alrededor de la lectura y los libros".

http://www.bibliotecanacional.gov.co/rnbp/noticias/ finalistas-del-premio-nacional

En la segunda convocatoria (2015) el acento recayó en los servicios bibliotecarios, su impacto en las comunidades y las estrategias para convertirse en escenario de encuentro y participación, espacio de disfrute cultural y de acceso a la información y al conocimiento. La ganadora fue la Biblioteca Clara Inés Campos Perdomo de San Vicente del Caguán, en el departamento del Caquetá. Se destacaron sus estrategias para contribuir al fortalecimiento de la identidad regional, a través de espacios de formación y promoción de producciones literarias locales que se constituyen en la voz de la comunidad en zonas de conflicto. 
http://www.bibliotecanacional.gov.co/rnbp/noticias/unabiblioteca-para-construir-la-paz

La bibliotecaria Lizeth-Paola Amezquita ha convertido la biblioteca en el epicentro para la recuperación de la memoria histórica de una región que en la mente de los colombianos está relacionada con la famosa zona de distinción, hace ya 13 años:

"Cuando empezamos teníamos dos o tres visitas diarias a la biblioteca, por lo que nos dimos cuenta que debíamos salir de las instalaciones e iniciar una campaña con la población y las visitas rurales, para que la gente sintiera más cercana la biblioteca, se apropiara de ella" (...).

Para ello se aliaron a la Casa de la Cultura. Además, empezaron a desarrollar un proceso de memoria histórica, pues no podían pensar en cambiar la imagen de San Vicente del Caguán sin antes empezar un proceso de identidad y apropiación por parte de sus pobladores.

“Nos encontramos con un fenómeno donde los jóvenes salían a ciudades como Florencia para sacar la cédula. Se avergonzaban que en el documento apareciera San Vicente del Caguán, pero también temían que al mostrar la cédula la gente los discriminara. De ahí comenzamos un proceso de memoria local para que desde los propios pobladores se identifique que San Vicente del Caguán va mucho más allá de haber sido una zona de distensión, porque jamás pidió o se le preguntó si quería serlo". http://www.bibliotecanacional.gov.co/rnbp/noticias/ biblioteca-publica-de-san-vicente-del-caguan-ganadora
En la tercera convocatoria (2016) priman los criterios de modelos de gestión, consolidación de servicios y programas bibliotecarios, experiencias exitosas y posicionamiento en sus comunidades; a la fecha no se conocen los resultados.

\section{La BP es un lugar privilegiado para con- solidar una cultura de la paz}

Si bien las experiencias descritas evidencian los procesos de transformación social que viven las comunidades como consecuencia de las acciones de la BP, existen muchas más, en mayor y menor rango, que buscan fortalecer el capital social de las comunidades desde la lectura del texto, pero también del contexto, la creación de contenidos locales, la convivencia y el encuentro ciudadano, la reconstrucción del tejido social, el aprendizaje individual y colectivo y la apropiación y uso de las TIC. Experiencias que dinamizan la función y misión de las BP, las declaran como espacios públicos vivos, que son y pertenecen a todos y que contribuyen a generar capital social a partir de su apuesta por la inclusión, el diálogo, la reconstrucción del tejido social y la recuperación y sistematización de la memoria local.

\section{Trayectorias y puntos de llegada}

Al constituirse las BP en escenario para el encuentro de la comunidad y en uno de los pocos espacios públicos gratuitos y abiertos al público que brinda diversidad de opciones de lectura, información, formación, y aún de ocio, su trabajo se vuelve estratégico en las políticas contra la exclusión social. En este contexto el reto es seguir generando valor y posicionando la BP como lugar vital, zona de distensión, de convivencia, de diálogo y como factor clave para la recuperación de la memoria histórica.

El acceso a la cultura, la recreación, la educación y la información posibilita encontrar otras formas de vida y de expresión distintas a las generadas por la violencia; la participación en las actividades culturales, recreativas y educativas brinda otras opciones de vida, abre nuevos horizontes, refuerza la autoestima, devuelve la esperanza, ayuda a resarcir el dolor y, muy especialmente, promueve la justicia social, la seguridad, la negociación de conflictos; en síntesis, contribuye a crear y consolidar una cultura de paz. En esta línea, las experiencias colombianas muestran cómo la BP pasa de un "deber ser" a un "ser": institución flexible, capaz de reorientar su trabajo en la formulación de estrategias para atender las trasformaciones sociales de la 
comunidad: ha logrado reinventarse y afrontar los cambios que el posconflicto demanda, al hacer de la BP un lugar de:

- inclusión y cohesión: donde se recreen los derechos humanos, sociales, políticos, económicos y culturales; se genere espacio y contenido para la diversidad e interculturalidad como fundamento de reconciliación, convivencia y paz. Por igual, que ofrezca oportunidades para el acceso igualitario a los medios de producción, salud, educación y cultura;

- diálogo: se estimula la convivencia y la participación entre grupos diferentes; se propicia la resolución de conflictos por medio de la negociación con estrategias de acogida, pertenencia, intercambios y encuentros;

- memoria local: se facilita la recuperación y sistematización de contenidos locales que permitan que la memoria pueda narrarse, que la identidad y las realidades de las comunidades puedan verse reflejadas, reconocidas, recordadas y resignificadas; la memoria local como una forma de resistencia.

Las BP como servicio público, agentes de cohesión social e instrumentos para la consolidación de la ciudadanía, existen para remover barreras en el ejercicio de la libertad y el disfrute de oportunidades, acciones que serán ejercidas por las comunidades más vulnerables y los ciudadanos menos afortunados en la distribución de la riqueza. Para lograrlo es necesario que la apuesta se oriente hacia la formación de bibliotecarios y promotores de lectura, escritura y oralidad de alto nivel, para enfrentar la complejidad de la paz y la conflictividad social. Que en ellas el bibliotecario se reinvente cada día, aúne esfuerzos, asuma riesgos, emprenda iniciativas y sueñe; este enfoque le exige habilidades de mediación, coordinación y organización de una biblioteca pública, para reafirmar su vocación de semillero de diálogo, convivencia, cohesión y memoria, donde germine la paz.

Finalmente, cada BP actual moldea su trabajo para que en medio del conflicto, de las carencias y necesidades de la comunidad, pueda abrirse como una "zona de distensión" que tienda puentes y ayude a la movilidad social, contribuya a neutralizar el conflicto social con la disposición de sus espacios para ejercitar la inclusión y la cohesión social, el diálogo y la memoria local, a partir de procesos formativos extraescolares. Se trata de una biblioteca viva integrada en las realidades sociales, de y para la comunidad, que propende por hacer realidad la interculturalidad, multiculturalidad, solidaridad, tolerancia, convivencia, construcción y reconstrucción de memoria y de tejido social, la formación a lo largo de la vida. Por ello la BP es un lugar privilegiado para consolidar una cultura de la paz.

\section{Notas}

1. Colombia tiene 47.870 .823 habitantes, distribuidos en 32 departamentos - unidad territorial de primer nivel en la división político-administrativa- y 1.123 municipios - unidad territorial de segundo nivel- donde el $100 \%$ de los municipios cuenta como mínimo con una biblioteca pública, categorizada de acuerdo con sus recursos físicos, de personal y tecnológicos: grandes, medianas y pequeñas.

http://www.dane.gov.co

2. Según la Encuesta de Consumo Cultural del DANE (2014) los ciudadanos que leen en Colombia consumen 4,2 libros al año; el índice de lectura está en 1,9. La meta del PNLB es llegar al 3,2.

\section{Bibliografía}

Arias, Diego (2015). “Los procesos de paz reunidos en nueve tomos: La 'Biblioteca de la paz' presenta la historia completa de los intentos de paz en el país". El tiempo, 28 de julio.

http://www.eltiempo.com/entretenimiento/musica-ylibros/biblioteca-de-la-paz-serie-de-libros-sobre-el-procesode-paz/16163655

CCP (Consejo de Cooperación Bibliotecaria, 2013). Prospectiva 2020. Las diez áreas que más van a cambiar en nuestras bibliotecas en los próximos años. Grupo Estratégico para el Estudio de la Prospectiva sobre la Biblioteca en el Nuevo Entorno Informacional y Social.

http://www.ccbiblio.es/wp-content/uploads/Estudio_ prospectiva_2020.pdf

DANE (2014). Encuesta de consumo cultural 2014: principales resultados. Bogotá.

http://www.dane.gov.co/files/investigaciones/eccultulral/ presentacion_ecc_2014.pdf

DANE (2016). Población de Colombia hoy. http://www.dane.gov.co

Jaramillo, Orlanda (2010). “La biblioteca pública, un lugar para la formación ciudadana: referentes metodológicos del proceso de investigación". Revista interamericana de bibliotecología, v. 33, n. 2, pp. 287-313.

http://www.redalyc.org/articulo.oa?id=179015630001

Jaramillo, Orlanda; Quiroz, Ruth-Elena (2013). "La educación social dinamizadora de prácticas ciudadanas en la biblioteca pública". Educação \& sociedade, v. 34, n. 122, pp. 139-154.

https://doi.org/10.1590/S0101-73302013000100008

McDermott, Caroline (2010). Desarrollo humano y las bibliotecas públicas de Colombia. Bogotá: Universidad de los Andes.

Meneses-Tello, Felipe (2013). "Bibliotecas y sociedad: el paradigma social de la biblioteca pública”. Investigación bibliotecológica, v. 27, n. 61, pp. 157-173.

http://www.revistas.unam.mx/index.php/ibi/article/ download/42818/38893

Red Nacional de Bibliotecas Públicas (2014). Diagnóstico Nacional de la Red Nacional de Bibliotecas Públicas. Bogotá: Ministerio de Cultura.

https://issuu.com/proyectotic/docs/diag_nacional_rnbp/0

Red Nacional de Bibliotecas Públicas. ¿Qué es la Red Nacional de Bibliotecas Públicas?

http://www.bibliotecanacional.gov.co/rnbp/que-es-la-rnbp

Sánchez-García, Sandra; Yubero, Santiago (2016). "Papel socioeducativo de las bibliotecas públicas: nuevos perfiles profesionales para nuevos tiempos". El profesional de la información, v. 25, n. 2, pp. 226-236.

https://doi.org/10.3145/epi.2016.mar.09

Unesco (1994). Manifiesto de la Unesco en favor de las bibliotecas pública. Paris: Unesco.

http://www.unesco.org/webworld/libraries/manifestos/ libraman_es.html 